Hot Topics on Vertebral Osteomyelitis from the International Society of Antimicrobial Chemotherapy

Kordo Saeed, Silvano Esposito, Tiziana Ascione,

Matteo Bassetti, Eric Bonnet, Alessia Carnelutti, Monica Chan , David Chien LYE, Nicholas Cortes, Matthew Dryden,

Shelanah Fernando, Thomas Gottlieb , Ian Gould, Karolin Hijazi , Simona Madonia, Pasquale Pagliano, Paul S Pottinger , John Segreti , Anna Maria Spera, on behalf of ISAC Skin, Bone and Joint Infection Working Group

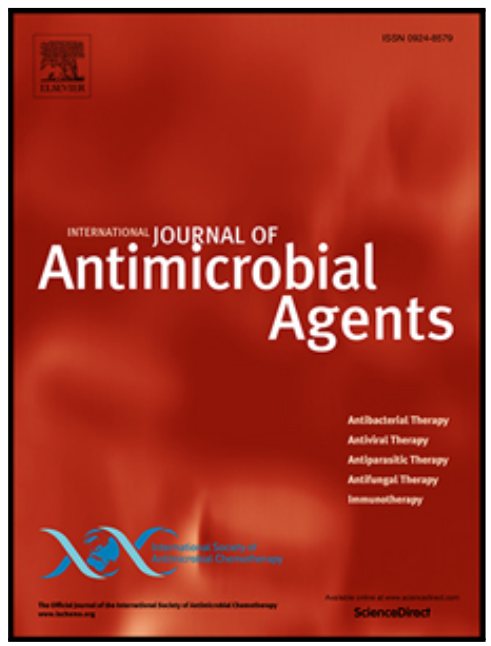

PII:

DOI:

Reference:

S0924-8579(19)30166-9

https://doi.org/10.1016/j.jiantimicag.2019.06.013

ANTAGE 5735

To appear in:

International Journal of Antimicrobial Agents

Received date:

15 April 2019

Accepted date:

10 June 2019

Please cite this article as: Kordo Saeed, Silvano Esposito, Tiziana Ascione, Matteo Bassetti, Eric Bonnet, Alessia Carnelutti, Monica Chan, David Chien LYE, Nicholas Cortes, Matthew Dryden, Shelanah Fernando, Thomas Gottlieb, lan Gould, Karolin Hijazi , Simona Madonia , Pasquale Pagliano, Paul S Pottinger, John Segreti, Anna Maria Spera, on behalf of ISAC Skin, Bone and Joint Infection Working Group, Hot Topics on Vertebral Osteomyelitis from the International Society of Antimicrobial Chemotherapy, International Journal of Antimicrobial Agents (2019), doi: https://doi.org/10.1016/j.ijantimicag.2019.06.013

This is a PDF file of an unedited manuscript that has been accepted for publication. As a service to our customers we are providing this early version of the manuscript. The manuscript will undergo copyediting, typesetting, and review of the resulting proof before it is published in its final form. Please note that during the production process errors may be discovered which could affect the content, and all legal disclaimers that apply to the journal pertain. 


\section{Hot Topics on Vertebral Osteomyelitis from the International Society of}

\section{Antimicrobial Chemotherapy}

Kordo Saeed ${ }^{1}$, Silvano Esposito ${ }^{2}$, Tiziana Ascione ${ }^{3}$, Matteo Bassetti ${ }^{4}$, Eric Bonnet ${ }^{5}$, Alessia Carnelutti ${ }^{4}$, Monica Chan ${ }^{6}$, David Chien LYE ${ }^{7}$, Nicholas Cortes ${ }^{1,8}$, Matthew Dryden ${ }^{1}$, Shelanah Fernando ${ }^{9}$, Thomas Gottlieb $^{10}$, Ian Gould ${ }^{11}$, Karolin Hijazi ${ }^{12}$, Simona Madonia ${ }^{2}$, Pasquale Pagliano ${ }^{3}$, Paul S Pottinger ${ }^{13}$, John Segreti ${ }^{14}$, Anna Maria Spera ${ }^{2}$ on behalf of ISAC Skin, Bone and Joint Infection Working Group.

1. Hampshire Hospitals NHS Foundation Trust, UK; University of Southampton Medical School, UK

2. Department of Infectious Disease, University Hospital San Giovanni di Dio e Ruggi d'Aragona, Salerno, Italy

3. Department of infectious diseases, AORN dei Colli, Naples - Italy

4. Infectious Diseases Clinic, Department of Medicine University of Udine and Azienda Sanitaria Universitaria Integrata di Udine, Udine, Italy

5. Department of Infectious Diseases, Joseph Ducuing Hospital et Clinique Pasteur, Toulouse, France.

6. Department of Infectious Diseases, Tan Tock Seng Hospital, National Centre for Infectious Diseases, Singapore

7. Tan Tock Seng Hospital; National Centre for Infectious Diseases; Yong Loo Lin School of Medicine; Lee Kong Chian School of Medicine; Singapore

8. Gibraltar Health Authority, Gibraltar, UK.

9. Department of Microbiology and Infectious Diseases, Concord Hospital, NSW Australia

10. Department of Microbiology and Infectious Diseases, Concord Hospital, NSW Australia and Department of Medicine University of Sydney 
11. Department of Medical Microbiology, Aberdeen Royal Infirmary, Aberdeen, UK

12. Institute of Dentistry, School of Medicine, Medical Sciences \& Nutrition, University of Aberdeen, Aberdeen, UK

13. Department of Medicine, Division of Allergy \& Infectious Diseases, University of Washington

14. Division of Infectious Diseases, Rush University Medical Center, Chicago, IL.

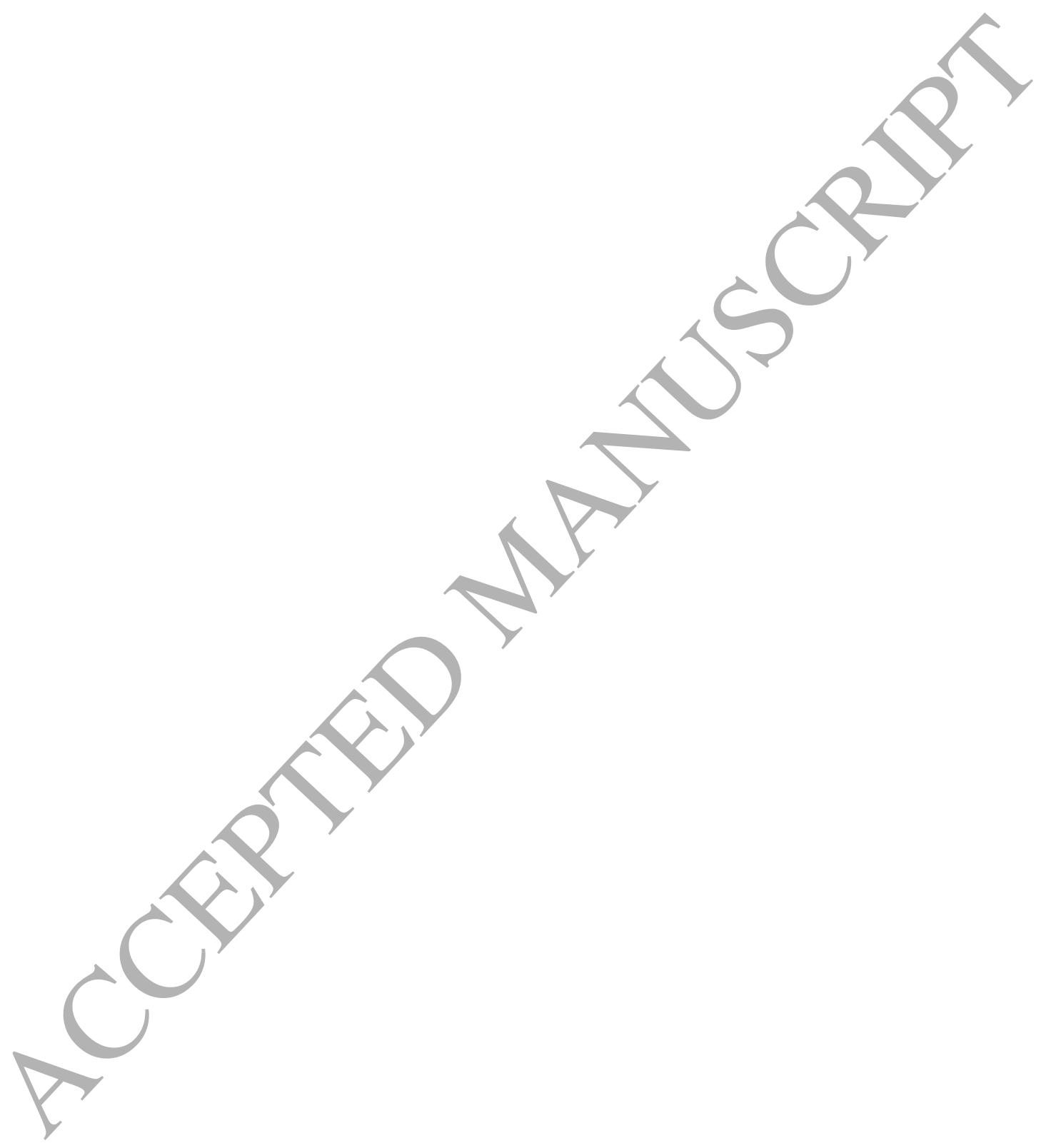




\section{Summary:}

Vertebral osteomyelitis (VO), also known as spondylodiscitis, describes infections of the vertebrae and intervertebral discs. Discitis describes infection limited to the intervertebral discs; in clinical practice both discitis and VO can be regarded as different stages of a single entity. VO can be caused by bacteria, fungi and parasites The incidence of VO is increasing globally representing $3-5 \%$ of all osteomyelitis with an estimated incidence ranging from 4 to 24 per million per year. Increasing incidence has been attributed to a combination of improved diagnostics, increased healthcare associated infections, haemodialysis, indwelling catheters, intravenous drug use, spinal instrumentation, immunocompromised hosts and an ageing popúlation [1].

If left untreated, VO can lead to irreversible spinal cord injury, deformity, neurologic deficits, septicaemia, and mortality (mortality rates ranges $4 \%-29 \%$ ). VO is typically treated with antibiotics, but up to $40 \%$ to $50 \%$ of VO patients may eventúally require surgical intervention [1-3] Despite advances in diagnostic modalities, medical and surgical care, there are still many controversial areas with regards to both diagnostic and therapeutic strategies in VO. In this review a number of 'hot topics' on VO were selected and reviewed by members of the Skin, Soft Tissue and Bone Infections Working Group of the International Society of Antimicrobial Chemotherapy (ISAC). This group includes international scientists, microbiology and infectious diseases clinicians and academics, whose aim is to advance the education and the science of infection management. This paper is an in-depth review of the current literature, providing a summary of the various aspects of VO and expert opinions and insights from the authors' own experience, highlighting areas for future study and research.

Key words:

Brucella, vertebral osteomyelitis, spondylodiscitis, Spinal Tuberculosis, fungal infections 


\section{A Clinical findings of vertebral osteomyelitis}

Vertebral osteomyelitis (VO) is clinically characterized by pain along the spinal area affected with or without fever. Focal back or neck pain is relatively common; occurring in most of cases, but up to $15 \%$ of patients may have no back pain. The pain can be acute or gradual in onset, progressing and worsening over several weeks to even months. Pain is typically exacerbated by physical activity, percussion of the affected area and more noticeable at night. The mean duration of symptoms is 48 +/- 40 days. Rarely a mass or spinal deformity may be clinically visible. Spinal deformities, such as kyphosis and gibbus deformity, are more frequent when the etiology is tubercular [1,4-6].

Radicular pain may radiate to the chest, abdomen, leg, scrotum, groin and perineum. Spine movements are often limited due to localized spinal pain and muscle spasm. Extension of infections posteriorly into the epidural space, leads to worsening back pain with radiculopathy and motor weakness and sensory changes and potential paralysis [7].

Physical examination can reveal signs of psoas abscess (e.g. flank pain and pain with hip extension) and neurologic signs in the lower limbs, and palpation for distended bladder. Abscess of cervical spine can be characterized by cervical rigidity, dysphagia or torticollis. Abscesses of the lumbar spine can spread through the ischiatic foramen and involve gluteus muscles. When lower lumbosacral roots are involved the "cauda syndrome" can appear. Sinus formation can be the result of a longstanding unrecognized infection [8].

Symptoms can be more non-specific in children: irritability, limping gait, hip pain, rejection to crawl, sit or walk, even abdominal pain and incontinence may also be present. Crucial signs include loss of lumbar lordosis and lower back movements; neurological deficits are unusual.

Fever is much less frequent than back pain: it occurs in only about half of patients. It is uncommon in mycobacterial, brucellar, or fungal VO and may be masked in patients on therapy with analgesics with antipyretic effects. However fever is common in older children with VO. Cervical infections, 
tuberculosis VO and late diagnosis are often associated with systemic symptoms of weight loss, weakness and anorexia $[1,4,5]$.

\section{B Imaging for vertebral osteomyelitis.}

Plain radiographs are often normal in the early phases, abnormal findings are usually not apparent before three weeks or more after the onset of symptoms. Frequent lesions include lysis of two contiguous vertebral bodies with collapse of the intervening disc space [9].

CT findings of VO appear earlier than those seen on plain radiographs. Main abnormalities include end plate irregularities. CT is especially useful for detecting bony sequestra and soft tissue abscesses, and to exclude epidural abscesses [10]. CT scan can also be useful for guiding vertebral biopsies. CT remains the first-line alternative test when MRI is not available or feasible [1].

MRI is the most sensitive imaging test for confirming VO diagnosis [10]. Abnormalities are often detected earlier on MRI than on CT. Typical findings on MRI include i) decreased signal intensity in the vertebral bodies and disc and loss of endplate definition (T1-weighted images), ii) increased disc signal intensity and/or increased vertebral body signal intensity (T2-weighted images), iii) contrast enhancement of the vertebral body and disc. Multi-sequence sagittal MRI of the entire spine may be helpful in patients with known single-level spine infection [11]. Follow-up by imaging including MRI is not recommended routinely. It should be only proposed for patients whose clinical status has not improved at the planned time for discontinuation of antibiotics in order to evaluate for the presence of an abscess in need of drainage or to detect spinal instability amenable to surgical intervention [1].

\section{Radionuclide scanning [12]}

- Three-phase bone scintigraphy using labeled technetium is neither sensitive nor specific enough for the diagnosis of vertebral osteomyelitis. False negatives have been reported mainly in the elderly. Therefore, bone scanning should not be used routinely in this setting. 
- Gallium scan; Osteomyelitis is likely if there is greater tracer uptake in the gallium scan compared to the three-phase scan. Conversely, if the gallium scan is normal, then osteomyelitis is unlikely, regardless of the bone scan findings. The main limitation of gallium scans are that they take $48-72$ hours to complete, necessitating multiple visits to the nuclear medicine department.

- Labeled-leukocyte scan is not recommended for the diagnosis of vertebral osteomyelitis due to poor sensitivity and specificity values.

- Positron emission tomography (PET) scanning using 18-fluorodeoxyglucose (FDG), combined with CT (PET-CT); During recent years many studies investigating the potential role of fluoride oxyglucose-positron emission tomography/computed tomography (FDG-PET/TC) for the management of a wide spectrum of infectious diseases, including $\mathrm{VO}$, have been published, with promising results [13]. Current Infectious Diseases Society of America (IDSA) guidelines recommend the use of FDG-PET/TC for the diagnosis of spondylodiscitis only when MRI cannot be obtained (e.g. patients with implantable cardiac devices, cochlear implants, claustrophobia, or in case of MRI unavailability) [1]. Although MRI still represents the gold standard technique for the diagnosis of spondylodiscitis, higher sensitivity and specificity values have been recently reported for FDG-PET/TC compared with MRI (95\% and $88 \%$ for FDG-PET/TC and $85 \%$ and $66 \%$ for MRI, respectively) [14,15]. However, false positive have been attributed to tumor, degenerative spinal disease, and/or spinal implants. Moreover, FDG-PET/TC has been associated with a higher diagnostic value compared with MRI for the detection of early spondylodiscitis within two weeks after symptoms onset [16]. Another important advantage of FDG-PET/TC over MRI is represented by the identification of metastatic foci of infection, especially in patients with bacteremia, allowing a prompt source control [14]. Conversely, MRI is more sensitive than FDG-PET/TC for the evaluation of soft tissue involvement and for the identification of small epidural abscesses [14]. To note, FDG-PET/TC is useful not only for the diagnosis of pyogenic spondylodiscitis, but also when mycobacteria, fungi or Brucella are 
involved, with some data showing different uptake values according with the etiology of infection [13]. Specifically, higher uptake values have been reported in tuberculous spondylodiscitis compared with pyogenic one [17].

The evaluation of response to antibiotic treatment in patients with spondylodiscitis represents an investigational role for FDG-PET/TC [13]. Overall, a significant improvement of FDG-PET/TC uptake values after 6 weeks of an adequate antibiotic treatment has been reported in patients with spondylodiscitis, but few data specifically addressing this topic are available so far $[18,19]$. A residual persistence of FDG uptake has been frequently reported also after an adequate course of antibiotic treatment, and the most appropriate interpretation of these data is matter of debate. Particularly, the pattern of residual activity seems to be crucial to distinguish between a mechanically induced residual inflammation, characterized by FDG uptake confined to the margins of the disc, and active infection, with FDG uptake extended to bone and soft tissues[19].

\section{C Microbiologic investigations for vertebral osteomyelitis}

The corner stone for microbiologic diagnosis is specimen culture obtained from a biopsy. A microbiologic diagnosis should be obtained to target antimicrobial treatment. But in special circumstances including neurologic compromise and sepsis, prompt empiric antibiotic therapy is warranted. However in patients with clinical, biochemical and imaging studies suggesting Vo with positive blood cultures for S. aureus or S. lugdunensis, then biopsies are not shown to add additional diagnostic value [1].

Blood cultures (at least 2 sets, aerobic and anaerobic bottles) are recommended. The correlation of blood cultures with cultures results from biopsy has been shown to be high for $S$. aureus and $S$. lugdunensis, but not for other bacteria including Gram negative rods and coagulase negative staphylococci. Therefore, biopsies are warranted in such situations to confirm the identity of the 
causative agent. Performing blood cultures immediately following biopsy does not add any benefit [20]. In the setting of positive blood cultures for staphylococci (especially for S. aureus), streptococci and enterococci, clinicians should look for concurrent infective endocarditis especially in patients with valvular prosthesis or underlying valvular disease and/or new-onset heart failure [21]. Vertebral needle biopsy is usually guided by CT. But in patients for whom an immediate surgical intervention (see below) is warranted samples could be obtained via open procedure. Although open biopsy had a higher diagnostic yield than needle biopsy, a CT-guided needle biopsy of the affected bone and aspiration of abscess, if present, is proposed initially because it is less invasive [22]. The specimens should be sent for bacterial (aerobic and anaerobic), and, in some circumstances, fungal, and mycobacterial (see below) cultures and also for histologic examination and if necessary polymerase chain reaction. Retaining some sample (unfixed) is recommended in case of need for subsequent molecular diagnostic testing. If the patient is on antibiotic treatment it is usually recommended to stop it several days before performing the biopsy because prior antibiotic exposure is likely to reduce the yield. However a recent study suggests that the negative effect on microbiologic results of antibiotics administered prior to percutaneous and open biopsy cultures might be overestimated [23].

If cultures of blood and the needle aspirate are negative and the suspicion for vertebral osteomyelitis remains high performing a second biopsy is suggested [20]. Alternative investigations include percutaneous endoscopic discectomy and drainage (PEDD), or open excisional biopsy. Another alternative to be discussed is the initiation an empiric therapy [22].

\section{Additional tests}

- Nucleic acid amplification testing may be useful if initial aerobic and anaerobic cultures are negative in patients who have already been treated with antibiotics or are infected with 
fastidious microorganisms such as Mycobacterium tuberculosis or Coxiella burnetii.

Contamination with skin flora may lead to false positive results.

- Brucella serology should be performed in patients with clinical signs suggestive of brucellosis and/or exposed to a potential source and/or coming from endemic areas [1].

- Cultures on specific media for fungi or mycobacteria could be proposed for patients with epidemiologic or host risk factors [1].

- Interferon (IFN)- $\boldsymbol{\gamma}$-releasing assay may also be performed in patients originating or residing in endemic regions or having risk factors for tuberculosis (TB). Because this test has a high sensitivity and negative predictive value ( $91 \%$ and $95 \%$ respectively in a recent study), it could be useful for excluding a diagnosis of active TB vertebral osteomyelitis [24].

\section{D Role of Erythrocyte sedimentation rate (ESR) and C-reactive protein (CRP)}

\section{in vertebral osteomyelitis}

Erythrocyte sedimentation rate (ESR) and C-reactive protein (CRP) are two commonly measured blood markers in VO. In a retrospective series of 345 cases of proven pyogenic VO, of which $74 \%$ were bacteraemic, median CRP was $13.1 \mathrm{mg} / \mathrm{dL}$ (IQR 5.5-22.0) and ESR 77mm/hour (IQR 55-100) [25]. In another retrospective series of 440 native pyogenic VO not associated with surgery, of which $78 \%$ was bacteraemic, mean ESR was $68 \pm 31 \mathrm{~mm} /$ hour and CRP was $13.5 \pm 9.5 \mathrm{mg} / \mathrm{dL}$ [26].Similarly a study of 42 surgically treated pyogenic VO with bacteraemia in $31 \%$, the mean ESR was $42 \mathrm{~mm} /$ hour (range, 3-140) and CRP was $14 \mathrm{mg} / \mathrm{L}$ (range, 1-29) on admission [27]. CRP can be elevated too; in a retrospective study of 40 patients with VO with $43 \%$ bacteraemia, the mean ESR was $79 \pm 27 \mathrm{~mm} /$ hour and CRP $165 \pm 79 \mathrm{mg} / \mathrm{L}$ on admission [28]. Additionally in a series of 129 patients with pyogenic VO, median CRP was significantly higher in culture-positive versus culture-negative cases ( $207 \mathrm{mg} / \mathrm{dL}$ versus $54 \mathrm{mg} / \mathrm{dL}$ ) [29]. In patients with pyogenic VO, even if previously exposed to 
antibiotics, open surgical biopsy or needle biopsy and higher median CRP, male gender, bacteraemia were independently associated with tissue culture positivity, but not antibiotic-free duration $[30,31]$. ESR and CRP have some utility in predicting treatment failure and poor outcome. An elevated CRP is associated with longer term functional disability from VO. [25,32]. .

Among a series of 40 patients, mean ESR was $85 \mathrm{~mm} /$ hour (range, $40-145$ ) which gradually declined to a mean of $25 \mathrm{~mm} /$ hour by the end of antibiotic therapy and to $12 \mathrm{~mm} /$ hour by 16 weeks. By the end of antibiotic therapy, ESR decreased to $50 \%$ of pre-treatment value in $94 \%$, and by 16 weeks, nearly half had normal ESR [33]. More than 50\% reduction in ESR from pre-treatment value in the first month, has been shown to rarely associated with failed conservative treatment [34]. In a 5-year retrospective series of 111 patients with pyogenic VO, ESR was elevated in 95\% on admission. Twenty three of 24 patients (96\%) less than 60 years with $>25 \%$ reduction in ESR from pre-treatment value at 4 weeks did well without surgery. In contrast, 2 of 15 patients (13\%) with impaired immunity and no change in ESR versus 34 of 36 patients (94\%) with normal immunity and $>25 \%$ reduction in ESR responded without surgery [35]. Additionally in patients with VO and bacteraemia, CRP $\geq 100 \mathrm{mg} / \mathrm{L}$, age $\geq 60$ years and Charlson's comorbidity index $\geq 2$ were independently associated with in-hospital mortality [36]. Despite all of the above CRP and ESR are non-specific markers; low values do not exclude VO, and higher values must be interpreted within the clinical context and other investigations when available.

\section{A Bacterial Vertebral Osteomyelitis}

The epidemiology of the pyogenic native VO varies in different parts of the world according to different social, economic and geographical features: typical bacterial agents such as Staphylococcus aureus, streptococcal species, enteric bacteria, and other Gram-negative rods are the most common pyogenic pathogens identified in native VO $[1,37]$. Haematogenous spread remains the most common route of infection, preferentially affecting the lumbar (58\%), thoracic (30\%) and cervical 
(11\%) regions with multifocal involvement being relatively uncommon (4\%) [4]. Indeed, the global increased incidence of VO may be partly attributed to MRSA bacteraemias which have compounded the burden of S. aureus bacteraemia [38]. Direct inoculation can occur following spinal surgery, instrumentation, lumbar puncture or epidural procedures. Delay in diagnosis is common, with average time to diagnosis approximately 2-4 months and initial mis-diagnosis in one third [4].

The prevalence of Gram-positive bacteria ranges from 26 to 93\%: Staphylococcus aureus is the most common organism, Gram-negative bacilli are isolated less frequently (table 1). Bacterial VO is commonly monomicrobial, with Staphylococcus aureus being the predominant pathogen in $20-84 \%$ $[1,4]$. The diagnosis should be suspected in patients complaining of new localized neck or back pain with concomitant or recent history of $S$. aureus blood stream infection $[1,4]$ Blood cultures can be positive in up to $50 \%$ of cases of S. aureus native VO. Obtaining a positive blood culture for S. aureus can obviate an image-guided aspiration specimen in patients with clinical, laboratory, and radiologic findings suggestive of native VO [39]. In a national Danish study including 8739 patients with S. aureus bloodstream infection, $6 \%$ of them were associated to native $\mathrm{VO}$ and were found in patients older than 50 years old without any identified obvious entry source [40]. Continuous bacteremia with the same coagulase-negative staphylococci in nephropathic patients under chronic hemodialysis with suspected native $V O$ or in patients with infected intravascular devices may also obviate the aspiration biopsy $[41,42]$. Staphylococcus lugdunensis, which can often behave like $S$. aureus, has been associated with deep-seated infections [43]. Polymicrobial infection accounts for the $9 \%$ of the analyzed cases [37].

It is also important to consider the involvement of anaerobe species in non-pyogenic intervertebral disease. This area has received significant attention following a double-blind randomised clinical controlled trial showing the efficacy of a 100-day course of co-amoxiclav in the treatment of lower back pain and Modic type 1 changes.[44] Notwithstanding that the current evidence from clinical trials is insufficient to recommend such a long-term course of antibiotics for any group of patients 
with chronic low back pain, there is abundant evidence showing colonisation of degenerated and oedematous discs by low-virulence anaerobes, such as Cutibacterium acnes (formerly Propionibacterium acnes) and coagulase-negative staphylococci $[45,46]$ C. acnes can be part of the normal oral microbiota and it has been hypothesised that bloodstream invasion may occur during low-grade oral trauma events such as toothbrushing, which may be particularly relevant in the absence of other septic foci. Indeed vertebral osteomyelitis secondary to common oral infections has been reported [47]. In our own clinical experience we have encountered oral commensal' streptococci, such as Streptococcus mitis, as one of the bacterial causes of Vo (unpublished). Nonetheless the exact role of all commensal species in the pathophysiology of Modic changes and intervertebral disc degeneration is yet to be elucidated [46].

\section{B Epidemiology of brucella vertebral osteomyelitis}

Brucellar Vertebral Osteomyelitis ( $\mathrm{B} \vee \mathrm{O}$ ) is common in countries of the Mediterranean basin, Latin America, the Middle East, parts of Africa, and Western Asia where human Brucellosis is endemic $[1,48]$.

In a large retrospective Spanish study conducted between 1982 and 2005, 918 patients were identified with brucella infection, of which $10.4 \%$ had vertebral localization [49]. In Turkey, Mete et al reported 100 cases of native VO between 2000 and 2007 from a single center; 24 of them had Brucella species [50]. Al Soub et al. in Qatar reported a (10.7\%) incidence of BVO [51].

Grammatico et al, on the contrary, reported that BVO accounted for $0.7 \%$ of their cases in France [52]. While, Sakkas et al described the epidemiology of native VO, in a single center in central Greece from 2000-2007, Brucella etiology accounted for 34\% of the cases [53]. Brucella spp were isolated in the blood of $37.5 \%$ and in the bone marrow of $66.7 \%$ of patients with brucellar species infection. Ten of 11 patients had IgM and IgG anti-brucella antibodies and a positive Rose-Bengal reaction. Three 
patients had been diagnosed with brucellosis and treated 5 months ( 2 patients) and 12 months ( 1 patient) earlier. One of these patients had detectable IgG and IgA, but not IgM antibrucella antibodies and responded to treatment. Outside the United States, the Coombs test is commonly used for the diagnosis of brucellar native VO [araj]. Enzyme-linked immunosorbent assay (ELISA) has proven to be superior in complicated cases of brucellosis and might be of value in patients with brucellar NVO [54].

According to data reported by Colmenero, that lumbar and lumbosacral level are involved in $67 \%$ of their cases; multiple-level involvement was described as $0-9 \%$ of cases [49]. The IDSA recommends a total duration of 3 months of antimicrobial therapy for most patients with NVO due to Brucella spp [1]. The two most commonly used regimens include combination of streptomycin for 2-3 weeks and doxycycline for 3 months, or doxycycline and rifampin (both for 3 months). In a cohort of patients $20 \%$ experienced treatment failure, with no significant difference between patients treated with doxycycline-streptomycin and those treated with doxycycline-rifampin [49].

In conclusion, as previously reported the incidence of BVO is extremely variable in those countries where human brucellosis is highly endemic. Thus, patients with native vo from highly endemic countries, Brucella spp have to be considered in the differential diagnosis.

\section{C Fungal vertebral osteomyelitis}

Fungal native $\mathrm{VO}$ is uncommon, ranging from $0.5-1.6 \%$ in most large case series, and associated with immunosuppression [4] as well as with intravenous drug use. In a large systematic review of reported cases from 1970 to 2011 of Candida osteomyelitis, 105 of 207 cases afflicted the vertebra. The commonest symptoms were local pain, tenderness and erythema in $93 \%$ with fever only in $28 \%$. Common radiological features were bony erosion in $66 \%$, reduced intervertebral space in $42 \%$ and extension into soft tissues and epidural abscess in $23 \%$ respectively. Median white cell count was $10,100 / \mathrm{mm}^{3}$, ESR $92 \mathrm{~mm} /$ hour and CRP $12 \mathrm{mg} / \mathrm{dL}[55]$. 
In a larger case series and literature review of 65 cases of Candida VO, $61 \%$ was associated with candidaemia with a delay of 2-12 months in $70 \%$ between onset of candidaemia and diagnosis of Candida VO. The most common vertebral sites were lumbar in $61 \%$, lower thoracic $41 \%$ and multiple levels $15 \%$. C albicans was responsible in $61 \%, C$ tropicalis $23 \%$ and C glabrata $9 \%$. Antifungal therapy alone was used in $50 \%$, surgery alone $5 \%$, and combined antifungal therapy and surgery $45 \%$ [56].

A review of 41 cases of Aspergillus VO, immunocompromised status was found in $66 \%$. Median delay in diagnosis was 12 weeks. Back pain was noted in $54 \%$ and neurological compromise in $29 \%$. White cell count was $<11,000 / \mathrm{mm}^{3}$ in $13 / 18$ and ESR $>40 \mathrm{~mm} /$ hour in $16 / 20$. Lumbar vertebrae were affected in $54 \%$, thoracic vertebrae $46 \%$, and multiple levels $22 \%$. Aspergillus fumigatus was isolated in $71 \%, A$ nidulans and $A$ flavus in $7 \%$ each. Antifungal therapy alone was used in $29 \%$ and surgery in $71 \%$, with overall recovery of $68 \%$ [57]. Increasingly, Aspergillus VO was reported to occur in immunocompetent patients with a more recent case series and literature review of 44 cases with predisposing conditions in $84 \%$, presumed to be haematogenous in $62 \%$ and contiguous $30 \%$. Fever was reported in $20 \%$, back pain $93 \%$, and neurological compromise $41 \%$. Surgery was performed in $57 \%$, and cure with antifungal therapy and surgery was $69 \%$ and antifungal therapy alone $71 \%$ [58].

\section{D Tuberculous vertebral osteomyelitis (TBVO)}

Although uncommon in the western world, tuberculosis remains an important cause of spinal infection globally. Among patients with extrapulmonary tuberculosis, 10-15\% have skeletal involvement, of which the spine is the most commonly affected site in approximately $50 \%[59,60]$. In Europe and USA, bone and joint infections account for $2.2-4.7 \%$ of tuberculosis cases overall. In developed countries, the disease typically affects older persons above 50 years of age, reflecting perhaps reactivation of legacy, latent TB, contrasting with children and younger adults presenting with spinal tuberculosis in endemic countries $[59,60]$. 
Unlike pyogenic VO, clinical presentation is characteristically slow and insidious with duration of symptoms lasting from weeks to years, averaging 4 to 11 months prior to diagnosis. Consequently, late complications including vertebral destruction and spinal cord compression are not uncommon [5]. Chronic back pain is the most frequent complaint, usually localized to the site of involvement. In up to $61 \%$ of cases, back pain is the only symptom at presentation [59]. Constitutional symptoms, such as fever, weight loss and malaise, are present in only $20-30 \%$ of osteoarticular tuberculosis. Advanced neurological deficits such as paraplegia, tetraplegia and spinal deformities may occur in $22-76 \%[59,60]$. Over $50 \%$ of patients will have evidence of a paraspinal abscess at the time of presentation [61]. Concomitant or reported history pulmonary tuberculosis is present in $50-75 \%$ $[59,60]$. On occasion, M. tuberculosis vertebral osteomyelitis may present with slow extension of infection into the soft tissues and ligaments, a "cold abscess". There is a notable absence of pain and other classic signs of inflammation in these instances [59]

Magnetic resonance imaging is the preferred neuroimaging modality. Spinal tuberculosis can affect any level of the spine, with predilection for the thoracic, followed by lumbar then cervical region. Whole spine screening can assess for multifocal non-contiguous involvement present in $16.3 \%$ to $71.4 \%^{1-2}$. Characteristic radiological findings are: destruction of bony vertebral bodies with relative preservation of intervertebral disc space, disruption of endplates, involvement of anterior vertebral body with sparing of the posterior arch, presence of spinal deformities and smooth walled paravertebral "cold" abscesses ${ }^{1-2}$. Chest X-ray may detect concomitant pulmonary tuberculosis $[59,60]$. In mycobacterial vertebral osteomyelitis higher uptakes levels at FDG-PET were detected in in comparison with pyogenic spondylodiscitis. PET-CT use appeared useful in the disease follow-up after treatment initiation to guide duration [17].

The paucibacillary nature of TBVO makes microbiological diagnosis challenging. Sampling of infected bone for mycobacterial culture in addition to histologic analysis is the critical factor in establishing a definitive diagnosis. The diagnostic yield for a percutaneous aspirate or CT guided biopsy is in the 
range of $42-76 \%$, however, the yield varies with the nature of the procedure performed [62].

Where possible, core biopsies are preferable to fine-needle aspirates [63]. Open biopsies have a greater sensitivity, however, are more invasive, they are recommended where an initial biopsy has been unsuccessful.

Radiologically guided specimens should be sent for mycobacterial smear and culture, histology and molecular testing if available. Due to the lower bacterial burden, smear positivity for acid-fast bacilli remains low, up to $52 \%$. Mycobacterial culture is the gold standard and positive in up to $83 \%$ of cases, but results are often delayed. Nucleic Acid Amplification testing (NAAT) including polymerase chain reaction (PCR) allows rapid turnaround with reported high sensitivity and specificity. However, the sensitivity is increased where tissue microscopy is positive for mycobacteria and hence, the yield remains dependent on nature and quality of the sample, this may be limited in paucibacillary infection. Increasingly NAAT assays are able to identify resistance to rifampicin, and use of multiplex assays or whole genome sequencing may in future be able to identify other resistance markers which may obviate culture. Nonetheless, current limitations necessitate the ongoing use of conventional culture methods to obtain a phenotypic antimicrobial susceptibility.

Histopathology is a key component of the diagnostic algorithm and often provides the first indication of tuberculosis; histology is confirmatory in approximately $60 \%$ with findings of epithelioid cell granulomas (85\%), granular necrotic background (82\%), lymphocytic infiltrate (76\%) and multinucleated Langerhans giant cells $(55 \%)[64,65]$. However, it is noted that some biopsies may be falsely negative.

The peripheral white cell may often be normal. Gok et al. (2014) reported an elevated leukocyte count of $>10,000 / \mathrm{mm} 3$ in only $22 \%$ of patients compared with $47 \%$ of patients with pyogenic vertebral osteomyelitis [5]. Elevated ESR, CRP, normochromic normocytic anaemia and hypoalbuminaemia may be present. Tuberculin Skin tests (TST) and Interferon gamma release assays (IGRA) are not useful for a definitive diagnosis of TBVO. The tests may help identify patients at risk of 
infection when positive. When negative, these can be used as an adjunct in excluding a diagnosis of active tuberculosis. However, either test may be negative in patients with latent or active tuberculosis, in the elderly, and also in those who are immunocompromised or immune-suppressed for other reasons $[59,60]$. Concurrent HIV must be diagnosed and treated.

Unlike other manifestations of tuberculosis, the evidence base for the management of TBVO relies on observational studies, particularly in relation to treatment duration. For drug susceptible $M$. tuberculosis strains, standard tuberculosis therapy is used (isoniazid, rifampicin, ethambutol and pyrazinamide for the first two months), however, the duration of isoniazid and rifampicin is often prolonged in practice. Individualised treatment is common; the total duration of therapy is typically in the range of 12-18 months [59]. Shorter treatment courses of 6-9 months are as effective and successful as 18-month regimens in trials conducted by the Medical Research Council (MRC) Working Party on Tuberculosis of the Spine ${ }^{5}$. The British Thoracic Society, American Thoracic Society and World Health Organisation recommend 6 months, $6-9$ months and 9 months treatment respectively for spinal tuberculosis. Longer duration of therapy may be indicated in patients on regimens not containing rifampicin, extensive or advanced disease and multi drug resistant (MDR) TB. If there is evidence of central nervous system involvement, including an epidural abscess, preemptive use of steroids to prevent the development of a paradoxical inflammatory reaction should be considered.

In ambulatory patients, there was no additional benefit of surgical debridement with good response in $82-95 \%$ on medical treatment alone ${ }^{5}$. However, surgery would still be indicated in patients failing to respond to conservative therapy, new or worsening neurological complications and mechanical instability from vertebral destruction or kyphosis. Of note, it is estimated that surgical intervention may be required in around $16 \%$ of patients with MDR TB despite maximal pharmacological therapy [66]. In addition, a propensity for relapse in patients with undrained secondary psoas abscesses is described. Hence, percutaneous drainage should be considered early in patients with large collections and those who are judged to have a poor clinical response to antimicrobial therapy [61]. 
Response to therapy can be difficult to gauge; assessment relies on gradual improvement in clinical parameters including pain scores, mobility, increase in body weight, and recovery of neurological deficits [61]. Serial imaging performed within the first six months of treatment will often suggest disease progression; hence, progress MRIs are not useful in monitoring response to therapy. However, progress MRIs remain necessary in patients who do not demonstrate expected clinical improvement $[59,61]$.

\section{A Antibiotic treatment of Bacterial (Pyogenic) Vo}

Most published guidance has previously recommended $6-12$ weeks of typically intravenous antibiotic treatment for a pyogenic discitis. The 2015 IDSA guidance shortens this to 6 weeks, moreover emphasising the role for oral antibiotics with high oral bioavailablity in addition to intravenous options. These guidelines recommend the prompt initiation of an empiric antibiotic treatment in patients with VO only in patients with neurological compromise and when signs of sepsis or hemodynamic instability are present [1]. Otherwise, when clinical conditions are stable and no signs of neurological involvement are present, the prescription of an antibiotic treatment should be postponed aiming to achieve a microbiological diagnosis. A 6-week course of parenteral or highly bioavailable oral antimicrobial therapy is currently recommended for patients with pyogenic spondylodiscitis, with prolonged antibiotic courses suggested only when Brucella is involved [1]. Antibiotic options for the treatment of spondylodiscitis according with etiology are summarized in Table 2 .

Many new antimicrobials with specific activity against gram-positive pathogens, including resistant isolates, have been recently introduced in routine clinical practice. Among these, dalbavancin and tedizolid represent investigational and interesting options for the treatment of vertebral osteomyelitis. Dalbavancin is a new parenteral lipoglycopeptide and is characterized by a prolonged half-life, allowing a single-dose infusion for the treatment of skin and soft tissue infections [67]. Dalbavancin possess a good bone penetration and has been recently found to be effective and well 
tolerated at the dose of $1500 \mathrm{mg}$ on days 1 and 8 for the treatment of osteomyelitis in adults [68]. Tedizolid is a new oxazolidinone and is currently approved for the treatment of acute bacterial skin and soft tissue infections. Advantages of tedizolid versus linezolid are the longer in vivo half-life allowing a once daily administration and the lower risk of myelotoxicity and drug - drug interactions with selective serotonin reuptake inhibitors and other compounds with serotonergic activity [69]. A phase 2 study investigating tolerability, safety and efficacy of tedizolid for the treatment of bone and joint infections is currently recruiting (NCT030090459).

The vast majority of studies on antibiotic duration are retrospective, observational studies. A single prospective open labelled randomised trial from France in adults demonstrated non-inferiority of 6 weeks duration of antibiotic treatment duration for microbiologically confirmed (positive blood culture or disc biopsy) discitis compared to 12 weeks [70].. Notably a high proportion (44\%) of patients were treated with predominantly oral antibiotics; with $52 \%$ of patients overall receiving intravenous treatment for less than 14 days in a non-standardised fashion. However, the authors report non-inferiority of 6-week regimes in the elderly (aged $>75$ years); patients with immunocompromise or diabetes, endocarditis or neurology, possibly due to power limitations. Restrictions in patient selection which may limit generalisability of these results are: exclusion of patients with a vertebral implant; those with no microbiological confirmation and those with fungal, Brucella or mycobacterial infection. Authors report a higher risk of treatment failure, regardless of duration, in patients aged $>75 y$ rs and those with $S$. aureus infection.

In relation to this pathogen, a single cohort retrospective study of antibiotic treatment of methicillinsensitive Staphylococcus aureus discitis in adults from Sheffield, UK, found similar outcomes in those receiving $\leq 12$ weeks treatment compared to those receiving $>12$ weeks treatment, with no difference between those who received $\leq 4$ weeks or $>4$ weeks intravenous antibiotics [71]. Infection due to MRSA, undrained paravertebral or psoas abscesses and end-stage renal disease were identified as independent risk factors for recurrence in a retrospective study by Park et al., and a 
prolonged course of antibiotic treatment ( $\geq 8$ weeks) was associated with a lower risk of recurrence compared with standard treatment duration (6-8 weeks) among high-risk patients presenting at least one risk factor for recurrence [72].

A number of studies have provided evidence to support the use of oral antibiotics in treatment of VO. In the study by Bernard et al [70]a high proportion (44\%) of patients were treated with predominantly oral antibiotics; with $52 \%$ of patients overall receiving intravenous treatment for less than 14 days in a non-standardised fashion. The results from the OVIVA study [73], a recently published multi-centre, randomised, open-label trial of initial 6 week intravenous versus oral antibiotic in osteomyelitis and bone infection, including VO patients, found non-inferiority with oral treatment. A retrospective study in VO patients managed surgically [74] reported non-inferiority of short ( $<3$ week) intravenous antibiotic courses, followed by 4 week oral treatment versus long ( $>3$ week) intravenous antibiotic courses in patients with $\mathrm{VO}$ with a low risk of recurrence; this was not the case in those with high risk of recurrence (risk factors: paraspinal abscess and/or positive blood culture). There is also one, intriguing, Turkish study [75]reporting success with a shorter, 4-week, course of intravenous antibiotic combined with hyperbaric oxygen treatment in post-microsurgical discectomy discitis with no oral continuation treatment. However, there are study limitations in terms of low microbiological confirmation rate, lack of therapeutic drug monitoring and absence of a comparator group.

Although risk factors for treatment failure and recurrence are not well established and no algorithms for the identification of high-risk patients are currently available, prolonged antibiotic treatment courses might probably be considered case by case based on patient's risk factors, particularly when source control is not timely performed. 


\section{B Indications for surgery}

Surgical intervention is indicated when patients develop progressive loss of motorand/or

neurological functions, cauda equina syndrome, progressive deformities or spinal instability. Failure of antibiotic therapy, as evidenced by persistent pain or systemic inflammation/ infection may also lead to surgical intervention [1,2]. Surgical management consists of debridement of all purulent and granulation tissue, sequestered bone and bone that is compressing neural structures [3].

An area of controversy is the optimal timing of surgery that most benefits the patient. Prior studies report conflicting findings. While some authors have found an advantage to earlier surgical intervention, others have not been able to show benefit [3]. All these studies are limited. They are typically small, retrospective and from a single center.

A study by Segreto, et al. evaluated the outcomes of early (less than 24 hours) versus delayed surgical treatment of VO using a large nationwide inpatient database [3]. This study found that VO patients who underwent surgery after 24 hours of admission had higher likelihood of morbidity and mortality. Unfortunately, an analysis such as this using a large health care database is not able to identify or control for confounding variables.

The optimal approach for the surgical management of $\mathrm{VO}$ is controversial and guided by clinical judgment and the experience of the surgeon. While an anterior approach allows better exposure for debridement and reconstruction, it may be more technically difficult than a posterior approach. Also controversial is use of instrumentation in patients with active infection, the benefit of one-stage versus two-staged procedures, and the use of autograft versus allograft. 
Nonetheless, several studies suggest favorable outcomes and low recurrent infection rates using instrumentation in the surgical treatment of spinal infection [2].

In summary, surgical treatment options for VO are varied and the selection of approach and procedure should be tailored to the individual patient's circumstances, in the absence of high-quality evidence to make more specific recommendations.

\section{Conclusion}

The review highlights key points related to diagnostic and therapeutic aspects in VO as well as important areas for future studies and research as there are still many unknowns with regards to:

1) What is or are the most effective investigations/technique for spinal biopsies and impact of these on clinical outcome and cost effectiveness of therapy?

2) Is finding aetiology affects patient's outcome?

3) Is outcome better with or without surgery and what is the best time for surgery if this is clinically indicated?

High quality studies and trails to guide empirical and tailored therapy for specific aetiologies within the above generic guidance are recommended.

\section{Declarations}

Funding: Nothing to declare, authors carried on this work in their own time.

Competing Interests: Nothing to declare

Ethical Approval: Not applicable

\section{References}


[1] Berbari EF, Kanj SS, Kowalski TJ, Darouiche RO, Widmer AF, Schmitt SK, et al. 2015 Infectious Diseases Society of America (IDSA) Clinical Practice Guidelines for the Diagnosis and Treatment of Native Vertebral Osteomyelitis in Adults. Clin Infect Dis 2015;61:e26-46. doi:10.1093/cid/civ482.

[2] Boody BS, Tarazona DA, Vaccaro AR. Evaluation and Management of Pyogenic and Tubercular Spine Infections. Curr Rev Musculoskelet Med 2018;11:643-52. doi:10.1007/s12178-0189523-y.

[3] Segreto FA, Beyer GA, Grieco P, Horn SR, Bortz CA, Jalai CM, et al. Vertebral Osteomyelitis: A Comparison of Associated Outcomes in Early Versus Delayed Surgical Treatment. Int J Spine Surg 2018;12:703-12. doi:10.14444/5088.

[4] Gouliouris T, Aliyu SH, Brown NM. Spondylodiscitis: update on diagnosis and management. J Antimicrob Chemother 2010;65:iii11-iii24. doi:10.1093/jac/dkq303.

[5] GÖk ŞE, Kaptanoĝlu E, Çelikbaş A, ErgÖnül Ö, Baykam N, Eroĝlu M, et al. Vertebral osteomyelitis: clinical features and diagnosis. Clin Microbiol Infect 2014;20:1055-60. doi:10.1111/1469-0691.12653.

[6] Nickerson EK, Sinha R. Vertebral osteomyelitis in adults: an update. Br Med Bull 2016;117:121-38. doi:10.1093/bmb/ldw003.

[7] Gasbarrini AL, Bertoldi E, Mazzetti M, Fini L, Terzi S, Gonella F, et al. Clinical features, diagnostic and therapeutic approaches to haematogenous vertebral osteomyelitis. Eur Rev Med Pharmacol Sci n.d.;9:53-66.

[8] Fantoni M, Trecarichi EM, Rossi B, Mazzotta V, Di Giacomo G, Nasto LA, et al. Epidemiological and clinical features of pyogenic spondylodiscitis. Eur Rev Med Pharmacol Sci 2012;16 Suppl 2:2-7.

[9] Gold RH, Hawkins RA, Katz RD. Bacterial osteomyelitis: findings on plain radiography, CT, MR, and scintigraphy. Am J Roentgenol 1991;157:365-70. doi:10.2214/ajr.157.2.1853823.

[10] An HS, Seldomridge JA. Spinal Infections. Clin Orthop Relat Res 2006;443:27-33. 
doi:10.1097/01.blo.0000203452.36522.97.

[11] Cox M, Curtis B, Patel M, Babatunde V, Flanders AE. Utility of sagittal MR imaging of the whole spine in cases of known or suspected single-level spinal infection: Overkill or good clinical practice? Clin Imaging 2018;51:98-103. doi:10.1016/j.clinimag.2018.02.009.

[12] Censullo A, Vijayan T. Using Nuclear Medicine Imaging Wisely in Diagnosing Infectious Diseases. Open Forum Infect Dis 2017;4. doi:10.1093/ofid/ofx011.

[13] Bassetti M, Carnelutti A, Muser D, Righi E, Petrosillo N, Di Gregorio F, et al. 18FFluorodeoxyglucose positron emission tomography and infectious diseases. Curr Opin Infect Dis 2017;30:192-200. doi:10.1097/QCO.0000000000000354.

[14] Kouijzer IJE, Scheper H, de Rooy JWJ, Bloem JL, Janssen MJR, van den Hoven L, et al. The diagnostic value of 18F-FDG-PET/CT and MRI in suspected vertebral osteomyelitis - a prospective study. Eur J Nucl Med Mol Imaging 2018;45:798-805. doi:10.1007/s00259-0173912-0.

[15] Kim S-J, Pak K, Kim K, Lee JS. Comparing the Diagnostic Accuracies of F-18 FDG PET and MRI for the Detection of Spondylodiscitis. Spine (Phila Pa 1976) 2018:1. doi:10.1097/BRS.0000000000002861.

[16] Smids C, Kouijzer IJE, Vos FJ, Sprong T, Hosman AJF, de Rooy JWJ, et al. A comparison of the diagnostic value of MRI and 18F-FDG-PET/CT in suspected spondylodiscitis. Infection 2017;45:41-9. doi:10.1007/s15010-016-0914-y.

[17] Bassetti M, Merelli M, Di Gregorio F, Della Siega P, Screm M, Scarparo C, et al. Higher fluorine-18 fluorodeoxyglucose positron emission tomography (FDG-PET) uptake in tuberculous compared to bacterial spondylodiscitis. Skeletal Radiol 2017;46:777-83. doi:10.1007/s00256-017-2615-8.

[18] Niccoli Asabella A, luele F, Simone F, Fanelli M, Lavelli V, Ferrari C, et al. Role of (18)F-FDG $\mathrm{PET} / \mathrm{CT}$ in the evaluation of response to antibiotic therapy in patients affected by infectious spondylodiscitis. Hell J Nucl Med n.d.;18 Suppl 1:17-22. 
[19] Riccio SA, Chu AKM, Rabin HR, Kloiber R. Fluorodeoxyglucose Positron Emission Tomography/Computed Tomography Interpretation Criteria for Assessment of Antibiotic Treatment Response in Pyogenic Spine Infection. Can Assoc Radiol J 2015;66:145-52. doi:10.1016/j.carj.2014.08.004.

[20] Gras G, Buzele R, Parienti JJ, Debiais F, Dinh A, Dupon M, et al. Microbiological diagnosis of vertebral osteomyelitis: relevance of second percutaneous biopsy following initial negative biopsy and limited yield of post-biopsy blood cultures. Eur J Clin Microbiol Infect Dis 2014;33:371-5. doi:10.1007/s10096-013-1965-y.

[21] Murillo O, Grau I, Gomez-Junyent J, Cabrera C, Ribera A, Tubau F, et al. Endocarditis associated with vertebral osteomyelitis and septic arthritis of the axial skeleton. Infection 2018;46:245-51. doi:10.1007/s15010-018-1121-9.

[22] Lew DP, Waldvogel FA. Osteomyelitis. Lancet 2004;364:369-79. doi:10.1016/S01406736(04)16727-5.

[23] Saravolatz LD, Labalo V, Fishbain J, Szpunar S, Johnson LB. Lack of effect of antibiotics on biopsy culture results in vertebral osteomyelitis. Diagn Microbiol Infect Dis 2018;91:273-4. doi:10.1016/j.diagmicrobio.2018.02.017.

[24] Choi S, Jung KH, Son H-J, Lee SH, Hong JM, Kim MC, et al. Diagnostic usefulness of the QuantiFERON-TB gold in-tube test (QFT-GIT) for tuberculous vertebral osteomyelitis. Infect Dis (Auckl) 2018;50:346-51. doi:10.1080/23744235.2017.1410282.

[25] Lee Y-M, Cho O-H, Park SY, Moon C, Chong YP, Kim S-H, et al. Factors associated with sequelae after treatment of hematogenous pyogenic vertebral osteomyelitis. Diagn Microbiol Infect Dis 2018. doi:10.1016/j.diagmicrobio.2018.11.024.

[26] Kim UJ, Bae JY, Kim S-E, Kim C-J, Kang S-J, Jang H-C, et al. Comparison of pyogenic postoperative and native vertebral osteomyelitis. Spine J 2018. doi:10.1016/j.spinee.2018.11.012.

[27] Dimar JR, Carreon LY, Glassman SD, Campbell MJ, Hartman MJ, Johnson JR. Treatment of 
pyogenic vertebral osteomyelitis with anterior debridement and fusion followed by delayed posterior spinal fusion. Spine (Phila Pa 1976) 2004;29:326-32; discussion 332.

[28] Chelsom J, Solberg CO. Vertebral osteomyelitis at a Norwegian university hospital 1987-97: clinical features, laboratory findings and outcome. Scand J Infect Dis 1998;30:147-51.

[29] Chong BSW, Brereton CJ, Gordon A, Davis JS. Epidemiology, Microbiological Diagnosis, and Clinical Outcomes in Pyogenic Vertebral Osteomyelitis: A 10-year Retrospective Cohort Study. Open Forum Infect Dis 2018;5:ofy037. doi:10.1093/ofid/ofy037.

[30] Kim C-J, Kang S-J, Yoon D, Lee MJ, Kim M, Song K-H, et al. Factors Influencing Culture Positivity in Pyogenic Vertebral Osteomyelitis Patients with Prior Antibiotic Exposure 2015. doi:10.1128/AAC.04949-14.

[31] Kim C-J, Kang S-J, Choe PG, Park WB, Jang H-C, Jung S-1, et al. Which tissues are best for microbiological diagnosis in patients with pyogenic vertebral osteomyelitis undergoing needle biopsy? Clin Microbiol Infect 2015;21:931-5. doi:10.1016/j.cmi.2015.06.021.

[32] Lemaignen A, Ghout I, Dinh A, Gras G, Fantin B, Zarrouk V, et al. Characteristics of and risk factors for severe neurological deficit in patients with pyogenic vertebral osteomyelitis. Medicine (Baltimore) 2017;96:e6387. doi:10.1097/MD.0000000000006387.

[33] Osenbach RK, Hitchon PW, Menezes AH. Diagnosis and management of pyogenic vertebral osteomyelitis in adults. Surg Neurol 1990;33:266-75.

[34] Carragee EJ, Kim D, van der Vlugt T, Vittum D. The clinical use of erythrocyte sedimentation rate in pyogenic vertebral osteomyelitis. Spine (Phila Pa 1976) 1997;22:2089-93.

[35] Carragee EJ. Pyogenic vertebral osteomyelitis. J Bone Joint Surg Am 1997;79:874-80.

[36] Loibl M, Stoyanov L, Doenitz C, Brawanski A, Wiggermann P, Krutsch W, et al. Outcomerelated co-factors in 105 cases of vertebral osteomyelitis in a tertiary care hospital. Infection 2014;42:503-10. doi:10.1007/s15010-013-0582-0.

[37] Mylona E, Samarkos M, Kakalou E, Fanourgiakis P, Skoutelis A. Pyogenic Vertebral Osteomyelitis: A Systematic Review of Clinical Characteristics. Semin Arthritis Rheum 
2009;39:10-7. doi:10.1016/j.semarthrit.2008.03.002.

[38] Lawes T, Edwards B, López-Lozano J-M, Gould I. Trends in Staphylococcus aureus bacteraemia and impacts of infection control practices including universal MRSA admission screening in a hospital in Scotland, 2006-2010: retrospective cohort study and time-series intervention analysis. BMJ Open 2012;2:e000797. doi:10.1136/bmjopen-2011-000797.

[39] Corrah TW, Enoch DA, Aliyu SH, Lever AM. Bacteraemia and subsequent vertebral osteomyelitis: a retrospective review of 125 patients. QJM 2011;104:201-7. doi:10.1093/qjmed/hcq178.

[40] Jensen AG. Staphylococcus aureus bacteremia. Dan Med Bull 2003;50:423-38.

[41] Cervan AM, Colmenero J de D, Del Arco A, Villanueva F, Guerado E. Spondylodiscitis in patients under haemodyalisis. Int Orthop 2012;36:421-6. doi:10.1007/s00264-011-1433-1.

[42] Faria B, Canto Moreira N, Sousa TC, Pêgo C, Vidinha J, Garrido J, et al. Spondylodiscitis in hemodialysis patients: a case series. Clin Nephrol 2011;76:380-7.

[43] Greig JM, Wood MJ. Staphylococcus lugdunensis vertebral osteomyelitis. Clin Microbiol Infect 2003;9:1139-41.

[44] Albert HB, Sorensen JS, Christensen BS, Manniche C. Antibiotic treatment in patients with chronic low back pain and vertebral bone edema (Modic type 1 changes): a double-blind randomized clinical controlled trial of efficacy. Eur Spine J 2013;22:697-707. doi:10.1007/s00586-013-2675-y.

[45] Chen Z, Cao P, Zhou Z, Yuan Y, Jiao Y, Zheng Y. Overview: the role of Propionibacterium acnes in nonpyogenic intervertebral discs. Int Orthop 2016;40:1291-8. doi:10.1007/s00264-016$3115-5$.

[46] Tang G, Wang Z, Chen J, Zhang Z, Qian H, Chen Y. Latent infection of low-virulence anaerobic bacteria in degenerated lumbar intervertebral discs. BMC Musculoskelet Disord 2018;19:445. doi:10.1186/s12891-018-2373-3.

[47] Rubin MM, Sanfilippo RJ, Sadoff RS. Vertebral osteomyelitis secondary to an oral infection. J 
Oral Maxillofac Surg 1991;49:897-900.

[48] Memish ZA, Balkhy HH. Brucellosis and international travel. J Travel Med n.d.;11:49-55.

[49] Colmenero JD, Ruiz-Mesa JD, Plata A, Bermudez P, Martin-Rico P, Queipo-Ortuno MI, et al. Clinical Findings, Therapeutic Approach, and Outcome of Brucellar Vertebral Osteomyelitis. Clin Infect Dis 2008;46:426-33. doi:10.1086/525266.

[50] Mete B, Kurt C, Yilmaz MH, Ertan G, Ozaras R, Mert A, et al. Vertebral osteomyelitis: eight years' experience of 100 cases. Rheumatol Int 2012;32:3591-7. doi:10.1007/s00296-0112233-z.

[51] Al Soub H, Uwaydah AK, Hussain AH. Vertebral osteomyelitis in Qatar. Br J Clin Pract 1994;48:130-2.

[52] GRAMMATICO L, BARON S, RUSCH E, LEPAGE B, SURER N, DESENCLOS JC, et al. Epidemiology of vertebral osteomyelitis (VO) in France: analysis of hospital-discharge data 2002-2003. Epidemiol Infect 2008;136:653-60. doi:10.1017/S0950268807008850.

[53] Sakkas LI, Davas EM, Kapsalaki E, Boulbou M, Makaritsis K, Alexiou I, et al. Hematogenous Spinal Infection in Central Greece. Spine (Phila Pa 1976) 2009;34:E513-8. doi:10.1097/BRS.0b013e3181a9897e.

[54] Araj GF, Kattar MM, Fattouh LG, Bajakian KO, Kobeissi SA. Evaluation of the PANBIO Brucella Immunoglobulin G (IgG) and IgM Enzyme-Linked Immunosorbent Assays for Diagnosis of Human Brucellosis. Clin Vaccine Immunol 2005;12:1334-5. doi:10.1128/CDLI.12.11.13341335.2005.

[55] Gamaletsou MN, Kontoyiannis DP, Sipsas N V., Moriyama B, Alexander E, Roilides E, et al. Candida Osteomyelitis: Analysis of 207 Pediatric and Adult Cases (1970-2011). Clin Infect Dis 2012;55:1338-51. doi:10.1093/cid/cis660.

[56] Hendrickx L, Van Wijngaerden E, Samson I, Peetermans WE. Candidal Vertebral Osteomyelitis: Report of 6 Patients, and a Review. Clin Infect Dis 2001;32:527-33. doi:10.1086/318714. 
[57] Vinas FC, King PK, Diaz FG. Spinal Aspergillus Osteomyelitis. Clin Infect Dis 1999;28:1223-9. doi:10.1086/514774.

[58] Nicolle A, de la Blanchardière A, Bonhomme J, Hamon M, Leclercq R, Hitier M. Aspergillus vertebral osteomyelitis in immunocompetent subjects: case report and review of the literature. Infection 2013;41:833-40. doi:10.1007/s15010-013-0463-6.

[59] Garg RK, Somvanshi DS. Spinal tuberculosis: A review. J Spinal Cord Med 2011;34:440-54. doi:10.1179/2045772311Y.0000000023.

[60] Jain A, Rajasekaran S. Tuberculosis of the spine. Indian J Orthop 2012;46:127. doi:10.4103/0019-5413.93671.

[61] Maron R, Levine D, Dobbs TE, Geisler WM. Two Cases of Pott Disease Associated With Bilateral Psoas Abscesses. Spine (Phila Pa 1976) 2006;31:E561-4. doi:10.1097/01.brs.0000225998.99872.7f.

[62] Colmenero JD, Jim nez-Mej as ME, Reguera JM, Palomino-Nic s J, Ruiz-Mesa JD, M rquez-Rivas J, et al. Tuberculous vertebralosteomyelitis in the new millennium: still a diagnostic and therapeutic challenge. Eur J Clin Microbiol Infect Dis 2004;23:477-83. doi:10.1007/s10096-004-1148-y

[63] Colmenero JD, Ruiz-Mesa JD, Sanjuan-Jimenez R, Sobrino B, Morata P. Establishing the diagnosis of tuberculous vertebral osteomyelitis. Eur Spine J 2013;22:579-86. doi:10.1007/s00586-012-2348-2.

[64] Mondal A. Cytological diagnosis of vertebral tuberculosis with fine-needle aspiration biopsy. J Bone Joint Surg Am 1994;76:181-4.

[65] Francis IM, Das DK, Luthra UK, Sheikh Z, Sheikh M, Bashir M. Value of radiologically guided fine needle aspiration cytology (FNAC) in the diagnosis of spinal tuberculosis: a study of 29 cases. Cytopathology 1999;10:390-401.

[66] Rasouli MR, Mirkoohi M, Vaccaro AR, Yarandi KK, Rahimi-Movaghar V. Spinal Tuberculosis: Diagnosis and Management. Asian Spine J 2012;6:294. doi:10.4184/asj.2012.6.4.294. 
[67] Dunne MW, Puttagunta S, Giordano P, Krievins D, Zelasky M, Baldassarre J. A Randomized Clinical Trial of Single-Dose Versus Weekly Dalbavancin for Treatment of Acute Bacterial Skin and Skin Structure Infection. Clin Infect Dis 2016;62:545-51. doi:10.1093/cid/civ982.

[68] Rappo U, Puttagunta S, Shevchenko V, Shevchenko A, Jandourek A, Gonzalez PL, et al. Dalbavancin for the Treatment of Osteomyelitis in Adult Patients: A Randomized Clinical Trial of Efficacy and Safety. Open Forum Infect Dis 2019;6. doi:10.1093/ofid/ofy331.

[69] Shaw KJ, Barbachyn MR. The oxazolidinones: past, present, and future. Ann N Y Acad Sci 2011;1241:48-70. doi:10.1111/j.1749-6632.2011.06330.x.

[70] Bernard L, Dinh A, Ghout I, Simo D, Zeller V, Issartel B, et al. Antibiotic treatment for 6 weeks versus 12 weeks in patients with pyogenic vertebral osteomyelitis: an open-label, noninferiority, randomised, controlled trial. Lancet 2015;385:875-82. doi:10.1016/S01406736(14)61233-2.

[71] Locke T, Kell ME, Bhattacharyya D, Cole AA, Chapman ALN. Spontaneous methicillin-sensitive Staphylococcus aureus spondylodiscitis - Short course antibiotic therapy may be adequate: Evidence from a single centre cohort. J Infect Public Health 2014;7:44-9. doi:10.1016/j.jiph.2013.08.001.

[72] Park K-H, Cho O-H, Lee JH, Park JS, Ryu KN, Park SY, et al. Optimal Duration of Antibiotic Therapy in Patients With Hematogenous Vertebral Osteomyelitis at Low Risk and High Risk of Recurrence. Clin Infect Dis 2016;62:1262-9. doi:10.1093/cid/ciw098.

[73] Lipsky BA, Hughes HC, Bose D, Kümin M, Scarborough C, Matthews PC, et al. Oral versus Intravenous Antibiotics for Bone and Joint Infection. N Engl J Med 2019;380:425-36. doi:10.1056/NEJMoa1710926.

[74] Li Y-D, Wong C-B, Tsai T-T, Lai P-L, Niu C-C, Chen L-H, et al. Appropriate duration of postsurgical intravenous antibiotic therapy for pyogenic spondylodiscitis. BMC Infect Dis 2018;18:468. doi:10.1186/s12879-018-3377-1.

[75] Kutlay M, Colak A, Simsek H, Yildiz S, Topuz K, Kaya S, et al. Antibiotic and hyperbaric oxygen 
therapy in the management of post-operative discitis. Undersea Hyperb Med n.d.;35:427-40.

Table 1. Comparative features and incidence of bacterial causes of vertebral osteomyelitis

\begin{tabular}{|l|l|l|}
\hline Microbiology* & Incidence & Route of infection \\
\hline Staphylococcus aureus & $20 \%-84 \%$ & $\begin{array}{l}\text { Most common pathogen. 1.7\% to 6\% blood stream infection } \\
\text { complicated by vertebral osteomyelitis }\end{array}$ \\
\hline $\begin{array}{l}\text { Coagulase-negative } \\
\text { Staphylococci }\end{array}$ & $5 \%-16 \%$ & $\begin{array}{l}\text { Device related bacteraemia or direct inoculation in post- } \\
\text { operative infections }\end{array}$ \\
\hline $\begin{array}{l}\text { Streptococci } \\
\text { Enterococci }\end{array}$ & $5 \%-20 \%$ & $\begin{array}{l}\text { Haematogenous spread. Associated with infective } \\
\text { endocarditis in 26\% }\end{array}$ \\
\hline Enterobacteriaceae & $7 \%-33 \%$ & $\begin{array}{l}\text { Haematogenous spread from urinary tract infections in older } \\
\text { population. Common E. coli, Proteus, Klebsiella, } \\
\text { Enterobacter spp. }\end{array}$ \\
\hline Anaerobes & $<4 \%$ & $\begin{array}{l}\text { Contiguous spread from pelvic or intraabdominal foci. } \\
\text { Propionibacterium acnes direct inoculation from implants }\end{array}$ \\
\hline Polymicrobial & $<10 \%$ & Contiguous spread \\
\hline
\end{tabular}


Table 2: Antimicrobial treatment options for spondylodiscitis*

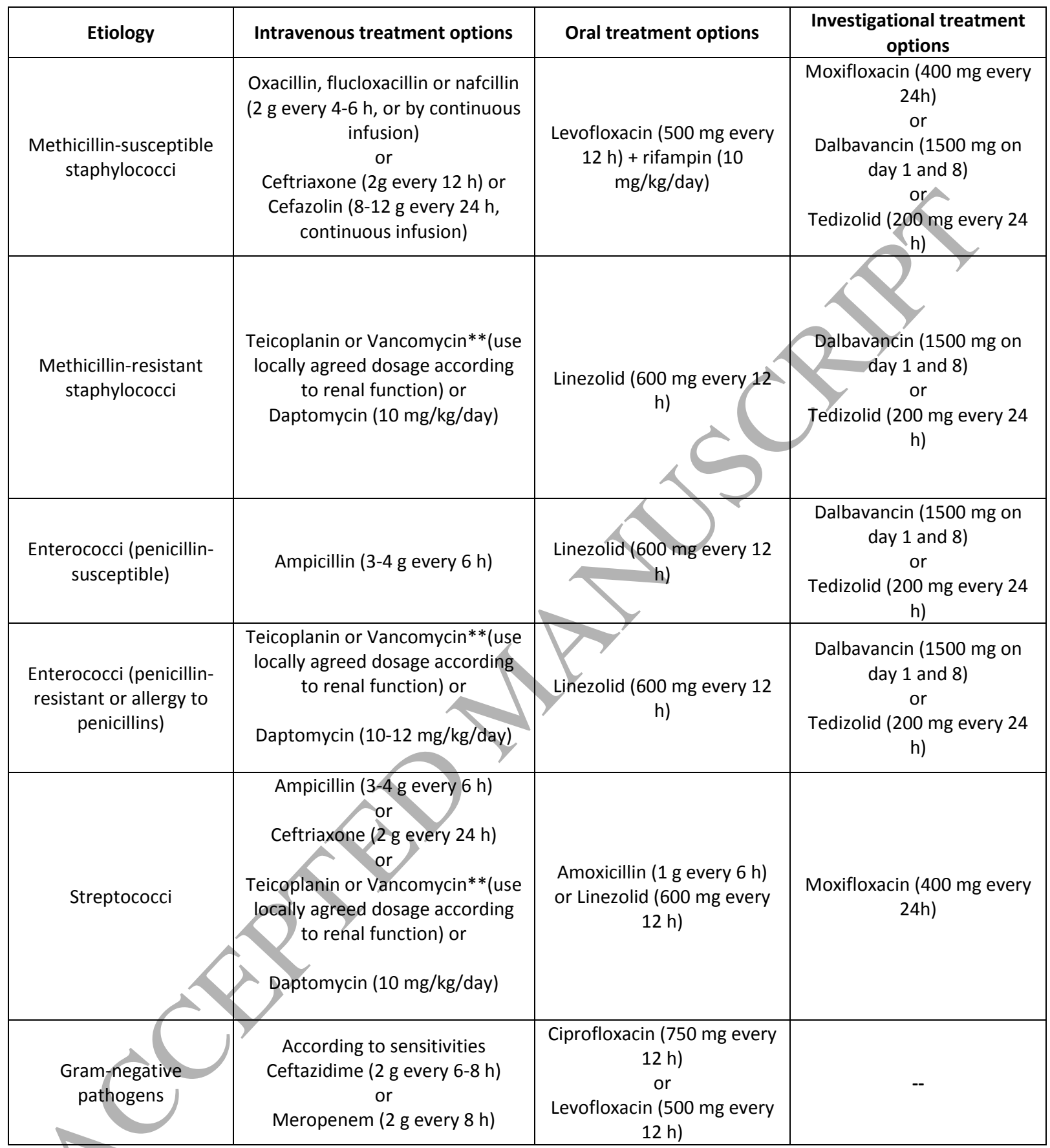

* Please follow local guidance and/ or local sensitivity pattern and/ or advice from your local infection specialists

**consider therapeutic drug monitoring, when available 